\title{
Regionalização e Constituição das Redes de Atenção à Saúde: Perspectiva de Gestores Municipais
}

\author{
Regionalization and Constitution of Health Care Networks: \\ Perspective of City Managers
}

\author{
Denise Finger $^{1}$ \\ Jeane Barros de Souza ${ }^{2}$ \\ Valéria Silvana Fagnello Madureira² \\ Daniela Savi Geremia ${ }^{2}$ \\ Larissa Hermes Thomas Tombini ${ }^{2}$
}

\section{RESUMO}

Objetivo: Analisar a percepção de gestores municipais de saúde sobre o processo de regionalização e constituição das Redes de atenção à saúde (RAS), identificando as dificuldades encontradas nas situações de referências e contrarreferência em saúde, na região do oeste catarinense. Metodologia: 0 estudo de campo, descritivo e exploratório, com abordagem qualitativa. A coleta de dados foi realizada com 13 gestores de saúde da região oeste do Estado de Santa Catarina. Os dados coletados foram analisados conforme a análise de conteúdo, proposta por Bardin (2011). Resultados: Vários aspectos sobre o processo de regionalização da saúde foram levantados pelos gestores entrevistados, como: insuficiência de serviços de saúde; necessidade de melhor organização dos serviços existentes na região; falta de referência e contrarreferência; principais demandas da região; fragilidade em relação aos recursos financeiros; convênios e pactuações entre os municípios e experiências exitosas dos municípios da região. Conclusão: Existem muitos obstáculos a serem superados e diversas mudanças a serem realizadas para alcançar a integralidade da assistência à saúde. No entanto, são inegáveis os avanços da regionalização do SUS, principalmente por meio do consórcio regional de municípios.

\section{DESCRITORES}

Gestor de Saúde. Município. Regionalização. Referência.

\begin{abstract}
Objective: analyze the perception of city health managers about the process of health regionalization and the constitution of the Redes de Atenção à Saúde (RAS), identifying the difficulties encountered in referral situations and on the western region of Santa Catarina. Methodology: the study consists of a field research, descriptive and exploratory, with a qualitative approach. Data collection was performed with 13 health managers from the western region of the Santa Catarina state. Moreover the data collected were analyzed according to the content analysis proposed by Bardin (2011). Results: several aspects about the process of health regionalization were raised by the managers interviewed, namely: insufficient services in the city and region; the need for better organization of health services in the region; Lack of reference and counter referral between services within the same city, as well as in the same health region; Main demands of the region; Fragility in relation to the financial resources allocated to the health care regionalization; Agreements and pacts between the cities and successful experiences of the cities of the region. Conclusion: there are still many obstacles to be overcome and a number of changes to be made to achieve comprehensive health care. However the SUS regionalization advances are undeniable, especially through the regional consortium of cities.
\end{abstract}

\section{DESCRIPTORS}

Health Manager. County. Regionalization. Reference.

\footnotetext{
${ }^{1}$ Enfermeira residente do Programa de Residência Multiprofissional em Saúde da Família da Universidade Federal de Santa Catarina, Florianópolis, Santa Catarina, Brasil.

${ }^{2}$ Docente do Curso de Enfermagem da Universidade Federal da Fronteira Sul - UFFS, Chapecó, Santa Catarina, Brasil.
} 
sistema único de saúde (SUS) estabeleceu as redes de atenção à saúde (RAS) para qualificar a atenção, ampliando o acesso e longitudinalidade do cuidado, em busca da integralidade na saúde ${ }^{1}$. As RAS são organizadas, tendo como porta de entrada a APS, que é responsável pela coordenação do cuidado e ordenação das redes, que visa a continuidade das ações e dos serviços de saúde de uma população, por meio de fluxos de comunicação interorganizacional para atingir uma relação integrada entre os elementos da sua estrutura operacional, interligando os pontos de atenção secundários e terciários, sistemas de apoio e a APS para alcançar a integralidade ${ }^{2,3}$.

Assim, foram adotadas as RAS para proporcionar consonância entre os princípios e diretrizes do SUS e o perfil epidemiológico brasileiro'. No entanto, a operacionalização das RAS no SUS ainda precisa se fortalecer, ficando evidente a necessidade de se realizar pesquisas científicas sobre o assunto ${ }^{4}$, a fim de contribuir na discussão de estratégias para sua efetivação ${ }^{1,3}$.

A conformação das RAS, assim como a regionalização do SUS, são importantes para a qualificação da saúde no país, pois buscam fortalecer a autonomia dos municípios, principalmente, daqueles de pequeno porte, que esbarram na falta de estrutura, com escassa arrecadação de recursos próprios para atender as necessidades de saúde da população. Cabe destacar que um dos maiores protagonistas nesse processo de fortalecimento da autonomia dos municípios e da organização entre estes de forma regionalizada, é o próprio gestor municipal de saúde, o qual possui grande responsabilidade frente à formulação de políticas públicas, as pactuações e as decisões sobre as ações implementadas.

Nesse âmbito, considerando a regionalização no SUS e o relevante papel que os gestores municipais de saúde possuem neste processo organizativo, questiona-se: qual é a percepção de gestores municipais de saúde sobre o processo de regionalização da saúde e configuração das RAS? Quais as dificuldades encontras nas situações de referência e contrareferência em saúde, na região do oeste catarinense?

Cabe destacar que as RAS precisam ser valorizadas para se tornarem efetivas na prática, a fim de superar as dificuldades na sua articulação e no planejamento ${ }^{5}$, juntamente com o princípio da regionalização da saúde, que são influenciadas diretamente pelas ações dos gestores municipais, justificando a relevância de estudar tal temática para reflexão e aprimoramento de políticas públicas e disseminação do conhecimento no setor, principalmente, em municípios de pequeno porte. Assim, o estudo teve como objetivo analisar a percepção dos gestores municipais de saúde sobre o processo de regionalização e configuração das RAS, identificando as dificuldades encontradas nas situações de referências e contrarreferência em saúde, na região do oeste catarinense.

\section{METODOLOGIA}

O estudo consiste em uma pesquisa de campo, descritiva e exploratória, com abordagem qualitativa. A coleta de dados foi realizada durante o segundo semestre de 2015 , nos municípios da Região Oeste do REstado de 
Santa Catarina, pertencentes às Secretarias de Desenvolvimento Regional (SDR) de Chapecó, Quilombo, Palmitos e Maravilha, que integram a Associação dos Municípios do Oeste de Santa Catarina (AMOSC). A amostra inicial totalizou 27 municípios: Águas de Chapecó, Águas Frias, Arvoredo, Caxambú do Sul, Chapecó, Cordilheira Alta, Coronel Freitas, Formosa do Sul, Guatambú, Iratí, Jardinópolis, Nova Erechim, Nova Itaberaba, Planalto Alegre, Pinhalzinho, Quilombo, Santiago do Sul, São Carlos, Serra Alta, Sul Brasil, União do Oeste, Caibi, Cunha Porã, Cunhataí, Mondaí, Palmitos e Riqueza.

As pesquisadoras entraram em contato, por telefone, com os gestores do setor saúde dos municípios da região do oeste catarinense, convidando-os para participar da pesquisa, sendo que 13 deles responderam positivamente. Assim, o estudo contou com a participação de 13 gestores dos seguintes municípios: Águas de Chapecó, Chapecó, Guatambú, Iratí, Nova Erechim, Planalto Alegre, Quilombo, Riqueza, Cunhataí, Formosa do Sul, Sul Brasil, União do Oeste e Pinhalzinho. Considerando a região da pesquisa, é importante salientar que todos os municípios são de pequeno porte (até 50.000 habitantes), exceto Chapecó, que se caracteriza como município de grande porte (100.001 a 900.000 habitantes), o qual é referência em serviços especializados de saúde para vários municípios da região. Portanto, a variação populacional dos municípios participantes do estudo está entre 1.949 e 209.553 habitantes, de acordo com a estimativa do censo $2010^{6}$. Assim, no momento da análise dos dados, tal característica foi considerada, a fim de buscar melhor compreender a realidade dos gestores municipais quanto ao processo de regionalização da saúde, na região do oeste catarinense.

Os dados foram coletados por meio de entrevistas semi-estruturadas, guiadas por um roteiro com sete perguntas que abordaram sobre: conceito de regionalização; papel da Secretaria Estadual de Saúde (SES) e Secretaria Municipal de Saúde (SMS) no processo de regionalização e constituição das RAS; distribuição da rede de serviços na região; principais necessidades da população; dificuldades e potencialidades da referência e contrarreferência na região; e os incentivos financeiros que estimulam a regionalização em saúde. As entrevistas foram realizadas em diferentes locais, conforme as possibilidades de cada participante, sendo gravadas em áudio e posteriormente transcritas.

Os dados coletados foram analisados conforme a análise de conteúdo, proposta por Bardin ${ }^{7}$. A análise dos dados primários foi realizada a partir do modelo analítico, desenvolvendo as seguintes: pré-análise; exploração do material ou codificação; tratamento dos resultados, inferência e interpretação ${ }^{7}$. Assim, emergiram duas categorias: 1) Percepção dos gestores municipais de saúde sobre o processo de regionalização; 2) Potencialidades e fragilidades da referência e contrarreferência no oeste catarinense.

O estudo iniciou-se somente após a aprovação do Comitê de Ética em Pesquisa com Seres Humanos, com Parecer $n^{\circ}$. 1.297.346, seguindo todas as preconizações da Resolução 466/2012, do Conselho Nacional de Saúde. Para garantir o anonimato dos participantes, optou-se em identificá-los pelo nome de rios que formam a vertente do interior 
de Santa Catarina, composta pela bacia hidrográfica do Rio Paraná e do Rio Iguaçu, pois assim como os rios menores se unem uns aos outros para formar as bacias hidrográficas, de tal maneira, os municípios se organizam em regiões pelas pactuações e formam as RAS, empoderando-se em busca de um objetivo em comum: fortalecer o SUS e garantir saúde universal e integral para a população.

\section{RESULTADOS E DISCUSSÕES}

Os 13 participantes do estudo ocupavam o cargo de gestor municipal de saúde entre seis meses e oito anos. Em relação à formação profissional e atuação anterior: dois eram profissionais da saúde, uma administradora, um contador, três agricultores, uma professora e os demais funcionários públicos que já atuavam em outras secretarias municipais.

Percepção dos gestores municipais de saúde sobre o processo de regionalização

A regionalização é abordada na Constituição Federal de 1988, a qual dispõe que as ações e serviços de saúde devem integrar uma rede regionalizada e hierarquizada ${ }^{7} \mathrm{Na}$ Lei Federal $n^{\circ} 8.080$, a regionalização é apresentada como um fator para a consolidação do princípio da descentralização político-administrativa ${ }^{8}$.

A Portaria $n^{\circ} 399$, de 22 de fevereiro de 2006, define como objetivos da regionalização: garantir que as ações e serviços de saúde sejam acessíveis, resolutivos e de qualidade; reduzir as desigualdades sociais e territoriais; proporcionar atenção à saúde integral, com acesso a todos os níveis de complexidade; fortificar o processo de descentralização através do apoio aos estados e municípios no exercício de gestores; racionalizar os gastos e otimizar os recursos destinados à saúde ${ }^{9}$. O Decreto $n^{\circ} .7 .508$, de 28 de Julho de 2011, apresenta o conceito de região de saúde, definindo-a como um espaço geográfico composto por municípios limítrofes, delimitado por características culturais, econômicas e sociais, além de redes de comunicação e de transporte compartilhados, objetivando integrar a organização, o planejamento e a execução de ações e serviços de saúde ${ }^{10}$.

Assim, considerando o conceito e os objetivos da regionalização, os gestores destacaram sua percepção sobre a regionalização:

Regionalização é definir os serviços, construir os serviços por região. Para você ter uma região de saúde, precisa ter os serviços mínimos necessários, se você não tiver o mínimo de serviços instalados, não pode ser uma região de saúde (Rio Peperi-Guaçú).

A regionalização são serviços que a gente tem, contrata, as referências que a gente tem na região. É quando a gente pensa, organiza o serviço, não pensar só pro meu município, entendeu?! É pensar de forma regionalizada, onde todos da região tenham acesso aos mesmos serviços (Rio Jangada).

Observou-se também que alguns gestores explanaram sobre o processo histórico da regionalização:

A regionalização já vem lá da Constituição de 88 como uma forma de organizar o serviço de saúde e para garantir os princípios constitucionais que são postos. Já ocorreram várias tentativas de regionalização, isso vem andando a passos muitos lentos e com algumas resistências às vezes, com 
passos mais largos em tempos, volta para trás noutro e agora com a implantação das redes de atenção eu tenho um pouco mais de crença que a gente consiga realmente por em prática efetivamente alguma coisa a mais na questão de regionalização (Rio Negro).

O processo de descentralização e regionalização no SUS teve muitos avanços e retrocessos, com incentivo à descentralização da gestão do SUS por meio da própria Lei $n^{\circ}$. 8.080, de 1990, a qual definiu as atribuições dos estados e municípios na saúde ${ }^{11}$. No entanto, além do conhecimento técnico por parte dos gestores, a estrutura dos serviços de saúde, o suporte logístico, de informação e comunicação, a qualificação de recursos humanos, a disponibilidade de recursos financeiros e principalmente a integração para pactuações de interesses comuns entre os municípios são essenciais para a consolidação da RAS.

Os entrevistados apontaram algumas dificuldades em relação à distribuição e organização dos serviços de referência dos municípios da região:

A gente dá conta da atenção básica, atenção primária. Qualquer referência especialidade é fora do município. Então a gente é atenção básica, NASF... (Rio Jacutinga).

Hoje, principalmente os municípios pequenos, eles têm muitas dificuldades, não dá conta de fazer os serviços. Nós dependemos de outros serviços em outros municípios (Rio Uruguai).

Além disso, a insuficiência de serviços na própria região também foi citada pelos participantes, o que obriga os municípios a procurar referências em centros maiores, principalmente a capital do estado, Florianópolis, que se localiza a $552 \mathrm{Km}$ do município de Chapecó-SC, como exemplo. Esses deslocamentos são um problema para a população devido à grande distância e, consequentemente, ao cansaço físico, o qual é ainda maior para pacientes debilitados:

[...] das demandas que a gente tem aqui, mas tem algumas coisas que não tem, que a gente tem que levar fora da região, levar a Florianópolis ou até em outros estados que é isso nossa maior preocupação (Rio Jacutinga).

[...] muitas coisas hoje pra nós conseguir é em Florianópolis. Então tem meses que é toda semana que a gente tem pacientes que vai pra lá pra tratamento [...] (Rio Uruguai).

A maioria dos municípios brasileiros, assim como os municípios participantes deste estudo, não são economicamente independentes para suprir todas as necessidades de saúde da população local. Por este motivo, é importante fortalecer as relações entre os municípios e as outras esferas de governo, buscando garantir o funcionamento da rede hierarquizada e regionalizada de atenção à saúde ${ }^{11}$

Ficou evidente que os gestores conhecem as limitações de seus municípios e da própria região em relação à disponibilização de serviços de saúde, mas também reconheceram a necessidade de melhor organização desses serviços existentes na região oeste catarinense, a fim de minimizar as dificuldades com a referência:

Eu vejo que é muito centralizado no município de Chapecó. Então, os outros municípios não procuram estabelecer serviços [...] Então penso que teria que ser mais distribuído, ter mais serviços em outros municípios (Rio Peperi-Guaçu). 
Apesar dos entrevistados citarem essas dificuldades em relação à distribuição dos serviços de referência na região, também referiram as potencialidades vivenciadas:

Nós, o município, temos algumas viagens pra Florianópolis por alguma coisa e outra, mas bem pouca [...] Então eu digo assim, hoje são poucos serviços de saúde que nós não temos em Chapecó né. Eu acho que nesse ponto melhorou muito e a tendência é sempre melhorar mais (Rio Canoas).

Agora que abriu aqui em Chapecó o hospital da criança, de pediatria, que antes era levado tudo pra Florianópolis, que era uma dificuldade grande, e agora vai ser aqui (oncologia pediátrica) (Rio Peixe).

A regionalização é um desafio que avança em direção a gestão compartilhada do SUS, o que possibilita a organização entre os municípios. O fato é que a municipalização no SUS enfrenta dificuldades quanto ao acesso à média e alta complexidade, principalmente dos municípios de pequeno porte, devido sua baixa capacidade instalada, ficando geralmente dependentes dos serviços de média e alta complexidade dos municípios maiores ${ }^{12}$. Isso pode ser observado nos depoimentos descritos acima, em que os próprios gestores citaram os avanços em relação à regionalização dos serviços de assistência a saúde no oeste catarinense. Da mesma forma, acredita-se que a articulação entre os municípios pode contribuir para a organização dos serviços de saúde em outras regiões do Brasil, superando as adversidades em cada realidade específica.
Potencialidades e fragilidades da referência e contrarreferência no oeste catarinense

A continuidade e integralidade da assistência à saúde têm esbarrado principalmente na falta de referência e contrarreferência entre os serviços, tanto dentro de um município, como na mesma região de saúde, necessitando de aprimoramento para qualificar a RAS. Essa dificuldade foi relatada pelos gestores:

Dentro do próprio município existe isso. É muito difícil ter a contrarreferência. O paciente é referenciado pro serviço e aí ele retorna sem nenhuma explicação, aí quem recebe o paciente não sabe... Não consegue dar continuidade. Isso tem que ser aprimorado. Se dentro do município acontece, imagina fora [...] (Rio Peperi-Guaçu).

Referência a gente até tem, o que a gente não tem é contrarreferência. Porque a gente manda o paciente, mas não traz retorno nenhum de volta pro município, então a gente fica a ver navios [...] ele (usuário) simplesmente, é como se ele tivesse desvinculado do município [...] (Rio Iguaçu).

Para enfrentar as deficiências da referência e da contrarreferência, é necessário criar estratégias de comunicação e reconhecimento mútuo entre as equipes e os diferentes serviços, com identificação das potencialidades e possíveis pactos de responsabilidades de cada parte, criando critérios de encaminhamento e de regresso dos usuários ${ }^{13,14}$. No entanto, existem dificuldades nesse diálogo entre os serviços do mesmo município e entre diferentes municípios, o que pode afetar diretamente a qualidade e a integralidade da assistência à saúde. 
Nota-se que a dificuldade em referenciar e contrareferenciar, por vezes, está associada às grandes demandas da região, principalmente em relação a algumas especialidades, como a ortopedia:

Mas o que nos preocupa bastante hoje aqui é a ortopedia[...] Não dá conta, é toda a região, se for analisar, acho que todos os municípios o problema é esse (Rio Uruguai).

As necessidades em ortopedia são comuns para a maioria dos municípios da região. A estrutura de serviços de saúde ainda é insuficiente e o Hospital Regional do Oeste, localizado em Chapecó-SC, é a referência em ortopedia para os municípios da região, atendendo 92 municípios da Região Oeste, 26 municípios do Paraná e do Rio Grande do Sul, num total de aproximadamente 1.000.000 habitantes ${ }^{15}$, sendo que esta grande demanda tem dificultado a referência e a contrarreferência. Essa fragilidade foi citada pelos gestores:

É Chapecó na questão da ortopedia, temos como referência, mas não tem a contrarreferência. [...] então o gargalo está ali, o problema está ali, teria que ser feito alguma coisa pra resolver a situação do Hospital Regional que é o nosso centro de referência hoje pra ortopedia... Não se tem nenhuma resolutividade (Rio Timbó).

Os relatos acima apontam que a demanda de especialidades e a insuficiente estrutura de serviços de saúde, é uma realidade em diferentes regiões do Brasil, o que tem dificultado o processo de referência e contrarreferrência, prejudicando a acessibilidade e a integralidade do cuidado ${ }^{12}$.

Além da ortopedia, os gestores também citaram outras demandas, como a on- cologia, a neurocirurgia, a cardiologia e as doenças mentais:

[...] a maior procura é a oncologia, ortopedia e neurocirurgia. Cardiologia e a área de pediatria, as doenças de saúde mental: álcool, drogas, e mesmo distúrbios mentais (Rio Peperi-Guaçu).

Nós temos aqui uma população que é da agricultura, então vem muita gente com LER DORT e doenças de pele, câncer de pele, essas coisas [...] (Rio Pelotas).

A grande demanda e a limitada oferta de serviços de saúde são observadas também em outro estudo, o qual mostra que essa dificuldade interfere no encaminhamento dos usuários. Os principais problemas identificados para a continuidade da assistência foram a inexistência ou precariedade da contrarreferência, a limitada oferta de consultas e exames e a má organização das atividades de regulação. Nesse sentido, os autores lembram que as condições de acesso aos serviços de saúde dependem, entre outros fatores, do dimensionamento correto da oferta em relação às necessidades de saúde e ao número de pessoas atendidas ${ }^{16}$.

Além da deficiência em relação à estrutura dos serviços da região, os gestores também citaram a fragilidade em relação aos recursos financeiros destinados à regionalização da assistência à saúde:

[...] falta investimento pra descentralizar serviços que nós continuamos ainda com grande parte dos serviços de média e alta complexidade centralizados na capital (Rio Negro).

Existem alguns incentivos das redes, mas, eu digo assim, insuficiente. Existem algumas redes bem estruturadas, a Urgência e Emergência teve um recurso 
bom [...] Tem realmente um pouco, mas não suficiente (Rio Peperi-Guaçu).

Mas aí assim, a conta sobra sempre pros municípios, se o governo federal não consegue mandar valor $\mathrm{X}$ e o estado não manda, o município tem que fazer (Rio Canoas).

Em relação ao financiamento das ações em saúde, a Lei n . 141-2012, estabelece os valores mínimos de responsabilidade de cada esfera, sendo que os municípios devem disponibilizar $15 \%$ da arrecadação tributária e os estados $12 \%{ }^{17}$. Os recursos provenientes da união foram alterados na Emenda Constitucional $n^{\circ} .86$, de março de 2015 , que define o percentual mínimo de investimentos em saúde pela União, o qual deverá alcançar, gradativamente, $15 \%$ da receita corrente líquida até $2018^{18}$.

Tais percentuais, apesar de serem importantes para a definição de recursos mínimos a serem aplicados em despesas com ações e serviços de saúde, não apresentam estabilidade financeira, pois estão relacionados com o crescimento econômico do ente federado, ou seja, pode variar intensamente de um ano para outro e não oferece sustentabilidade para as tomadas de decisões. Vale destacar que, além dos percentuais, a forma e os critérios para a destinação dos recursos financeiros é igualmente importante. Os incentivos financeiros do SUS devem ser adaptados às condições naturais, demográficas, epidemiológicas, sociais e culturas de cada região ${ }^{18}$.

Apesar da gestão dos serviços de saúde ser da competência das três esferas de governo, o financiamento foi e continua sendo um grande desafio para todos os en- volvidos nesse processo ${ }^{19}$. Isso pode ser observado no relato dos gestores, que abordaram sobre a sobrecarga dos municípios quanto às obrigações financeiras relacionadas à saúde.

As dificuldades citadas pelos gestores, como a grande demanda, estrutura insuficiente e o financiamento precário, deveriam ser motivos para promover a união e o trabaIho integrado entre os pequenos municípios. Todavia, de acordo com os entrevistados, ainda é um desafio a integração entre os municípios:

[...] mas ninguém tem esse comprometimento de ajudar, de facilitar, eu acho que todo mundo às vezes só pensa em si [...] e as coisas não deveriam ser assim, eu acho que é o comprometimento de cada secretaria, de cada secretário, de levar a sério a saúde como um todo, pensando em todos, não só no seu município (Rio Timbó).

Para a regionalização da atenção à saúde, deve-se observar as necessidades de saúde da população e da região, o que demanda reconfigurações das fronteiras, por meio de um território sanitário comum para garantir o cuidado integral ${ }^{20}$. No entanto, esse espaço regional de serviços de saúde condiciona-se à capacidade de organização e a pactuação entre os gestores (municipais, estaduais e federais), em um âmbito marcado por desigualdades na negociação, o que favorece a desarticulação e escassa cooperação intergovernamental ${ }^{21}$.

Além da tímida integração entre diferentes municípios, outro aspecto negativo levantado pelos gestores é a alta rotatividade de pessoas no cargo de Secretário Municipal de Saúde, o que também dificulta a criação 
de vínculos com outros municípios e, consequentemente, fragiliza a RAS da região:

[...] a rotatividade é muito grande de secretários e aquela pessoa que entra ali, ela não sabe trabalhar com gestão, ela não sabe trabalhar com financiamento, muito menos se pensar em regionalização, porque ela acha que o município tem que resolver tudo e não é assim. Ela precisa dos vizinhos pra resolver (Rio Iguaçu).

A alta rotatividade dos gestores, principalmente devido aos processos partidários e eleitorais, é uma fragilidade da gestão em saúde, o que gera descontinuidade nas ações e desmotivação dos profissionais e trabalhadores. Somado a isso, ainda existe o modelo de contratação temporária e a carga horária reduzida dos profissionais que atuam no setor, o que corrobora com a rotatividade, dificultando a criação de vínculo com a população, comprometendo a longitudinalidade e a continuidade do cuidado, como proposto pela RAS. Assim, fica evidente a necessidade de fortalecimento dos vínculos com a APS, sendo relevante o papel dos gestores para amenizar essa situação ${ }^{22}$.

Outra fragilidade encontrada foi a falta de ética de alguns profissionais da saúde:

[...] você tem um paciente que está 3 meses, 4 meses, 5 meses na fila esperando uma cirurgia, que essa cirurgia gratuita não acontece, daqui a pouco da a impressão de que se alguém oferecer valor $X$ pro profissional lá da ponta, a fila anda, as portas se abrem e acontece muito [...] o SUS é ótimo, só que eu tenho a impressão que no meio do caminho, pelo fato de que alguns profissionais não usarem da ética e do bom senso, acaba tendo alguns problemas e daí ele (SUS) não é 100\% (Rio Canoas).

De fato, a falta de ética e a corrupção estão presentes em diferentes cenários mundiais, sendo que na saúde isso não é diferente. No entanto, muitas vezes isso é mascarado pelo cargo ou posição social ocupado. A "falta de ética", citada pelo gestor, sugere a utilização de função ou cargo no serviço de saúde para transformar os atendimentos em concessão de favores, ferindo os princípios do SUS e as suas finalidades. Neste contexto, os profissionais precisam se responsabilizar para atingir os objetivos das RAS, sendo que a formação de recursos humanos é crucial para a efetividade da integração das redes, em busca de qualificar os profissionais para atuar como protagonistas no sistema de gestão das RAS, incluindo respeito, ética e solidariedade ${ }^{22}$

Apesar de tantas dificuldades e fragilidades citadas pelos gestores, eles também abordaram aspectos positivos em relação à RAS e a regionalização do SUS no oeste catarinense, ressaltando experiências exitosas:

[...] a gente tem uma renda, por exemplo, fixa que é o mínimo que a gente tem que ministrar em saúde que é $15 \%$ do que vem pro município e além disso, a gente recebe recursos, a gente pactua diversos programas, programa de próteses que a gente recebe um valor por mês, programa do PMAC, do NASF, são diversos programas que a gente recebe incentivos[...] (Rio Preto).

Nós utilizamos aqui no município o consórcio CIS AMOSC, que o que a gente não consegue suprir com SUS, digamos assim... Tem ainda a PPI (Programação Pactuada Integrada), que é um valor que a gente recebe pra fazer, então a gente as vezes acaba negociando alguma coisa $\mathrm{PPI}$, digamos assim, exames laboratoriais, exames de sangue, eu recebo um valor PPI, mas eu não utilizo, eu utilizo o consórcio CIS AMOSC, então eu consigo, final do ano, remanejar o valor desse pra outro, digamos, pra ressonância, que é uma 
coisa que eu utilizo mais...é bem completo esse consórcio, tem vários municípios que utilizam, então é muito bom, por que eu não sei o que a gente faria se não tivesse isso...(Rio Pelotas).

Apesar de muitas críticas em relação à falta de comprometimento dos municípios, alguns gestores citaram os bons resultados dos convênios e parcerias:

É, a gente busca discutir isso e às vezes dividir entre secretários nas reuniões da $\mathrm{CIR}$, ou dos colegiados da AMOSC, por exemplo, envolver os secretários em diversas câmaras técnicas, ou grupos condutores de alguma rede ou algum serviço [...] Eu acredito que dessa forma a gente consegue envolver e dividir um pouco, pra que todos participem, todos estejam envolvidos. Muitos preferem ficar de fora, preferem que outros façam, porque tem mais experiência, porque tem mais conhecimento... Mas eu acho que é bom todo mundo se envolver, porque daí todos acabam conhecendo como funcionam as redes, o sistema e tudo mais (Rio Jangada).

Além destes convênios e parcerias entre os municípios, vale também ressaltar o importante papel da Comissão Intergestores Regional (CIR), Comissão Intergestores Bipartite (CIB) e Comissão Intergestores Tripartite (CIT) na regionalização da assistência à saúde e a sua integralidade. Essas comissões também foram lembradas pelos gestores:

Os municípios estão organizados com a $\mathrm{CIB}$, tem CIR, tem SDR e tem AMOSC, então esses grupos são onde os municípios se reúnem e cada um discute as ações que estão sendo desenvolvidas, e nesses mesmos campos a gente tenta montar formas que a gente consegue montar uma ação que envolva todos os municípios pra ganhar divulgação, pra ganhar apreço, pra ter resultados, montar uma rede, pequenas redes de atendimento (Rio Canoinhas).

A gente planeja junto, a gente que nem eu citei antes nós, no nosso grupo de SDR nós temos seis municípios, a gente senta e discute junto, planeja junto, a gente faz reuniões, faz ata a partir dali a gente tem encontros na, aqui por exemplo quando tem de gestores o nosso grupo da AMOSC, então sempre tem troca de experiência pra tudo e nunca se perde o tempo, a viagem, quando você vem num encontro desse (Rio Preto).

As Comissões Intergestores (CIR, CIB e CIT) se caracterizam como espaços de pactuações entre os gestores do SUS, a fim de discutir, negociar e executar políticas de saúde, firmando parcerias e reforçando a regionalização das ações e dos serviços de saúde ${ }^{11}$. Considerando as parcerias entre os municípios de uma região e entre os municípios e as demais esferas, desvela-se o conceito de regionalização cooperativa, que reforça a necessidade de espaços que privilegiam associação entre os municípios e as ações solidárias ${ }^{23}$.

A realidade apresentada neste artigo se refere aos pequenos municípios, que perceberam na regionalização e na cooperação entre si, a oportunidade de qualificar e garantir a assistência integral à saúde para a sua população. Nesse sentido, percebe-se que apesar das dificuldades e entraves, a regionalização do SUS pode gerar resultados positivos quando existir comprometimento e compartilhamento de responsabilidades entre os envolvidos.

\section{CONCLUSÃO}

Diante das relações governamentais intermunicipais, os gestores municipais de 
saúde são atores essenciais nas pactuações para a regionalização das ações e dos serviços. A região oeste catarinense enfrenta algumas dificuldades, como: insuficiência de serviços de saúde nos municípios e na própria região; necessidade de melhor distribuição dos serviços existentes; falta de referência e contrarreferência dentro do município e entre diferentes municípios; alta demanda e estrutura insuficiente dos serviços de saúde da região; recursos financeiros insuficientes; falta de comprometimento de alguns municípios em relação à integralidade da assistência à saúde; alta rotatividade de pessoas que assumem o cargo de Secretário Municipal de Saúde; e a falta de ética de alguns profissionais da rede, que destorcem a real finalidade do SUS, transformando o que é um direito de todos, em privilégios para poucos aliados. Todos esses aspectos dificultam a regionalização no SUS e a constituição das redes de atenção à saúde e, consequentemente, resulta em prejuízos para a qualificação da assistência à saúde.

Mas apesar de tantas dificuldades, os gestores apontaram avanços em relação à saúde na região oeste catarinense, como: elevação do número de serviços especializados na região, diminuindo as viagens até a capital do estado; incentivos financeiros provenientes de programas, consórcios e pactuações; experiências positivas em relação à referência e contrarreferência com alguns serviços e municípios; parcerias realizadas entre municípios próximos; importante atuação das comissões intergestores na regionalização e organização das redes na região.

Portanto, percebe-se que ainda existem muitos obstáculos a serem superados e diversas mudanças a serem realizadas para alcançar a integralidade da assistência à saúde. No entanto, são inegáveis os avanços da regionalização do SUS, alcançados principalmente por meio do consórcio regional.

Como limitação do estudo cita-se a não abrangência de todos os gestores da região estudada, o que poderia trazer maiores informações e discussões sobre as ações de regionalização e constituição das RAS. Incentiva-se a continuidade de estudos abordando tal temática, em outras localidades do Brasil, a fim de ampliar as reflexões e contribuir para a qualificação da RAS em todo o território nacional.

\section{REFERÊNCIAS}

1. Brasil. Portaria № 4.279, de 30 de dezembro de 2010 . Estabelece diretrizes para a organização da Rede de Atenção à Saúde no âmbito do Sistema Único de Saúde (SUS). Brasília (DF): Ministério da Saúde; 2010.

2. Lamothe L, Sylvain C, Sit V. Multimorbidity and primary care: Emergence of new forms of network organization. Sante Publique. 2015; 27(Suppl.1):S129-35.

3. Vargas I, Mogollón-Pérez AS, De Paepe P, Ferreira da Silva MR, Unger JP, Vázquez ML. Barriers to healthcare coordination in market-based and decentralized public health systems: a qualitative study in healthcare networks of Colombia and Brazil. Health Policy Plan. 2016 31(6):736-48. 
4. Noronha JC. Redes integradas de cuidados e a pesquisa necessária. Divulg Saúde Debate. 2014; 52:50-53.

5. Maria José de Oliveira EvangelistaAlzira Maria D’Àvila Nery GuimarãesEliana Maria Ribeiro DouradoFabiana Loureiro Binda do ValeMaria Zélia Soares LinsMarco Antônio Bragança de MatosRaquel Beviláqua Matias da Paz Medeiros SilvaSimone Alexandra SchwartzO Planejamento e a construção das Redes de Atenção à Saúde no DF, Brasil Ciênc. Saúde Colet. 2019; 24(6) 27.

6. IBGE. Instituto Brasileiro de Geografia e Estatística. Censos Demográficos. Cidades, 2010.

7. Bardin, NL. Análise de Conteúdo. São Paulo; 2011.

8. Brasil. Constituição da República Federativa do Brasil, outubro de 1988.

9. Brasil. Lei Federal $n^{\circ} 8.080$, de 19 de Setembro de 1990. Dispõe sobre as condições para a promoção, proteção e recuperação da saúde, a organização e o funcionamento dos serviços correspondentes e dá outras providências. Casa Civil, Subchefia para Assuntos Jurídicos 1990; 19 Set.

10. Brasil. Decreto $n^{\circ} 7.508$, de 28 de Junho de 2011. Regulamenta a Lei $\mathrm{n}^{\circ} 8.080$, de 19 de setembro de 1990 , para dispor sobre a organização do Sistema Único de Saúde - SUS, o planejamento da saúde, a assistência à saúde e a articulação interfederativa, e dá outras providências. Casa Civil, Subchefia para Assuntos Jurídicos 2011; 28 Jun.

11. Sauter AMW,Girardon-Perlini NMO, Kopf ÁW. Política de regionalização da saúde: das normas operacionaisao pacto pela saúde. Rev Min Enferm. 2012; 16(2):265-274.

12. Medeiros CRG, Gerhardt TE. Evaluation of the Health Care Network in small towns from the perspective of management teams. Saúde Debate. 2015; 39(no. spe):160-170.

13. Aguiar ZN. SUS: Sistema Único de Saúde- antecedentes, percurso, perspectivas e desafios. 2.ed. São Paulo: Martinari; 2015.

14. Aires LCP, Santos EKA, Bruggemann OM, Backes MTS, Costa R. Referência e contrarreferência do bebê egresso da unidade neonatal no sistema de saúde: percepção de profissionais de saúde da Atenção Primária. Esc Anna Nery. 2017; 21(2):e20170028.

15. Hospital Regional do Oeste- HRO. Institucional. História. 2015.
16. Serra CG, Rodrigues PHA. Avaliação da referência e contrarreferência no Programa Saúde da Família na Região Metropolitana do Rio de Janeiro (RJ, Brasil). Ciência \& Saúde Coletiva. 2010; 15(3):3579-3586.

17. Brasil. Lei complementar $N^{\circ} 141$, de 13 de Janeiro de 2012.Regulamenta o $§ 3^{\circ}$ do art. 198 da Constituição Federal para dispor sobre os valores mínimos a serem aplicados anualmente pela União, Estados, Distrito Federal e Municípios em ações e serviços públicos de saúde; estabelece os critérios de rateio dos recursos de transferências para a saúde e as normas de fiscalização, avaliação e controle das despesas com saúde nas 3 (três) esferas de governo; revoga dispositivos das Leis $n^{\circ \mathrm{s}} 8.080$, de 19 de setembro de 1990, e 8.689, de 27 de julho de 1993; e dá outras providências. Casa Civil, Subchefia para Assuntos Jurídicos 2012, 13 Jan.

18. Brasil. Emenda Constitucional $n^{\circ} 86$, de 17 de Março de 2015. Altera os arts. 165, 166 e 198 da Constituição Federal, para tornar obrigatória a execução da programação orçamentária que especifica. Casa Civil, Subchefia para Assuntos Jurídicos 2015, 17 Mar.

19. Casanova AO, Cruz MM, Giovanella L, Alves GR, Cardoso GCP. Health care networks implementation and regional governance challenges in the Legal Amazon Region: an analysis of the QualiSUS-Rede Project. Ciênc Saúde Coletiva. 2017; 22(4):1209-1224.

20. Almeida PF, Santos AM, Santos VP, Silveira Filho RM Integração assistencial em região de saúde: paradoxo entre necessidades regionais e interesses locais. Saúde Soc 2016; 25:320-35

21. Albuquerque MV, Viana ALA, Lima LD, Ferreira MP Fusaro ER, lozzi FL, et al. Desigualdades regionais na saúde: mudanças observadas no Brasil de 2000 a 2016. Ciênc Saúde Colet 2017; 22:1055-64.

22. Peiter, $\mathrm{CC}$ et al . Redes de atenção à saúde: tendências da produção de conhecimento no Brasil. Esc. Anna Nery. 23(1):e20180214, 2019

23. Bousquat A et al. A atenção primária em regiões de saúde: política, estrutura e organização. Cad Saúde Pública. 2019; 35(2):e00099118, 2019.

\section{CORRESPONDÊNCIA}

Denise Finger

Avenida Mauro Ramos, 1250, Centro

Florianópolis, Santa Catarina, Brasil. CEP:88020-301.

E-mail: deni.finger@hotmail.com 